\title{
COVID-19 E MULHERES: UM ESTUDO SOBRE A DESIGUALDADE DE GÊNERO E A GOVERNANÇA FEMININA
}

\author{
COVID-19 AND WOMEN: A STUDY ON GENDER INEQUALITY AND FEMALE GOVERNANCE
}

\author{
Yhasmin Monteiro Pinto Moreno Leon* \\ Natália Gomes Martins de Oliveira***
}

\begin{abstract}
Resumo:
A pandemia da COVID-19 causou impactos em todo o mundo, afetando diversos países em diferentes níveis. Em muitos destes, questões antigas, relacionadas com disparidades de gênero, foram especialmente ampliadas, tais como violência doméstica, disparidades salariais, bem como a distribuição desigual do trabalho com base no papel de gênero. Contudo, apesar desse cenário, o contexto de crise social e econômica gerado pelo coronavírus mostrou-se também uma oportunidade para as mulheres demonstrarem a incoerência dos papéis de gênero, uma vez que as líderes femininas alcançaram melhores resultados em termos de taxas de morte e infecção quando comparadas com lideranças masculinas, sendo tratadas como exemplos de governança mundial no combate à pandemia. Dessa forma, partir de uma fundamentação adquirida por meio de uma pesquisa bibliográfica de característica qualitativa, o presente artigo buscou apurar a construção histórica de barreiras socioculturais que têm gerado tais desigualdades de gênero e excluído as mulheres de posições de poder e de tomada de decisão, a fim de constatar a incoerência dos estereótipos de gênero. Através da leitura de artigos acadêmicos, livros, reportagens e dados oficiais acerca do tema, o artigo constatou a efetividade de lideranças femininas no controle da pandemia em razão de fatores psicológicos e estratégicos presentes nos governos das mulheres, tais como um comportamento avesso ao risco associado a uma postura empática e sensível que transmitia confiança, tendo por resposta uma maior adesão social às medidas e políticas de confinamento adotadas.
\end{abstract}

Palavras-chave: COVID-19. Governança feminina. Estereótipos de gênero.

\begin{abstract}
:
The COVID-19 pandemic has had an impact worldwide, affecting several countries at different levels. In many of these, old issues, related to gender disparities, have been especially widened, such as domestic violence, wage disparities, as well as the unequal distribution of work based on gender role. However, despite this scenario, the context of the social and economic crisis generated by the coronavirus also proved to be an opportunity for women to demonstrate the inconsistency of gender roles, since female leaders achieved better results in terms of death and infection rates when compared to male leaders, being treated as examples of global governance in combating the pandemic. Thus, based on a foundation acquired through a qualitative
\end{abstract}

\footnotetext{
Graduanda em Direito pela Faculdade de Direito da Universidade de São Paulo e pela Université Jean Monnet, por meio do programa PITES - Partenariat International Triangulaire d'Enseignement Supérieur.

** Graduanda em Direito pela Faculdade de Direito da Universidade de São Paulo e pela Université Lumière Lyon 2, por meio do programa PITES - Partenariat International Triangulaire d’Enseignement Supérieur.
} 
bibliographic research, this article sought to investigate the historical construction of socio-cultural barriers that have generated such gender inequality and excluded women from positions of power and decision-making, in order to verify the inconsistency of gender stereotypes. By reading academic articles, books, reports and official data on the topic, the article found the effectiveness of female leaders in controlling the pandemic due to psychological and strategic factors present in women's governments, such as a behavior that is averse to the risk associated with an empathetic and sensitive posture that conveyed confidence, and could led to a greater social adherence to the measures and policies of social confinement.

Keywords: COVID-19. Female governance. Gender stereotypes.

\section{Metodologia}

O presente artigo foi desenvolvido a partir de uma fundamentação adquirida por meio de uma pesquisa bibliográfica de característica qualitativa. A pesquisa foi dividida em dois distintos momentos: no primeiro, buscou-se entender os fatores históricos relacionados à desigualdade de gênero, como a divisão sexual do trabalho e os papéis sociais de gênero, de forma que, para atingir esse objetivo, foram analisados artigos acadêmicos e livros acerca do tema. Em um segundo momento, a pesquisa voltou-se para o momento atual pelo qual o mundo está passando, qual seja, a vivência da pandemia da COVID-19, com a leitura de artigos acadêmicos e matérias jornalísticas sobre o tema. Buscou-se compreender os efeitos que a pandemia acarretou, até o presente momento, para as mulheres - em diferentes nichos, perspectivas e contextos sociais - e, ainda, no último tópico, empreendeu-se esforços para compreender as diferenças entre os mecanismos adotados por governantes do sexo masculino e feminino no combate à pandemia, com o objetivo de averiguar as razões por trás da constatação de que governos femininos obtiveram melhores resultados (lê-se, menos mortes), no enfrentamento a COVID-19, sendo esta última constatação feita a partir de dados oficiais liberados pelos governos e por entidades, além de artigos recentes que trataram da matéria.

Introdução

Durante muitos anos as mulheres se viram excluídas da esfera política e dos cargos de poder, pois, dentro de uma sistemática pautada pelas relações sociais de sexo, fora estipulada uma divisão sexual do trabalho que destinava às mulheres os papéis de cuidados com a casa e família, enquanto aos homens eram destinados os papéis mais valorizados, dentro de uma lógica capitalista - relacionados ao trabalho produtivo e intelectual. Foram necessários muitos anos de luta para que as mulheres pudessem adentrar nesses espaços, sendo sua escalada nessas esferas bastante árdua e custosa. 
Nos dias de hoje, o número de mulheres a ocupar cargos de destaque aumentou, todavia, ainda é possível notar uma enorme influência do fenômeno denominado como teto de vidro, o qual, em decorrência da propagação de estereótipos e padrões discriminatórios baseados em critérios exógenos e endógenos, pautados pelos papéis sociais de gênero, exclui as mulheres das mais altas esferas de poder e destaque em suas carreiras. A lógica organizacional do mercado de trabalho está, ainda, baseada em um padrão de governança e comando masculino, sendo que, dentre as barreiras invisíveis que se apresentam às mulheres para atingir os cargos de liderança, encontram-se: a dupla jornada de trabalho, a falta de infraestrutura adequada e uma cultura organizacional sexista, que compõem alguns dos pilares da desigualdade de gênero enraizada na estrutura social. Mesmo quando as mulheres conseguem superar as diversas barreiras sociais que encontram em seus caminhos e, consequentemente, alcançam posições de decisão e poder, tendem a permanecer por menos tempo nesses cargos que os homens e, ainda que ocupem os exatos mesmos cargos que eles, obtém uma menor remuneração.

Com a pandemia da COVID-19, muitas das problemáticas historicamente enfrentadas pelas mulheres tiveram um recrudescimento, tornando o problema ainda mais latente. Dentre as questões analisadas, constatou-se um aumento, em nível mundial, dos índices de violência doméstica em decorrência do isolamento social, que impôs a muitas mulheres um confinamento junto de seus agressores; um aumento da execução, pelas mulheres, de trabalhos domésticos e não remunerados, como os cuidados com crianças, idosos e enfermos; e um crescimento da taxa de desemprego entre as mulheres, que foram mais afetadas que os homens por serem a maioria da força de trabalho no setor informal e em áreas especialmente afetadas pela pandemia.

Porém, não apenas a pandemia afetou a vida de milhares de mulheres, como também as mulheres, no caso, governantes e chefes de estado, influenciaram de maneira notável no combate à pandemia da COVID-19. Aqui, não podem ser ignorados os resultados expressivos obtidos por Estados cujo governo fora exercido por uma mulher, nos quais o número de mortes em decorrência da pandemia, em comparação com governos masculinos, foi sensivelmente menor. Dentre os motivos para isso foram averiguados: maior empatia; a tendência à priorização da preservação da vida, e não da economia; e a tomada de decisões que expressaram uma maior cautela, já que agiram, em sua maioria, rapidamente, consultando profissionais especializados e determinando o lockdown ou a quarentena logo no início da pandemia.

A presente análise restringiu-se aos países da OCDE, uma vez que, se incorporados todos os países, seria impossível estabelecer um padrão com base em circunstâncias semelhantes, dado que países com governos ditatoriais e autoritários apresentam estratégias de governança totalmente distintas e contrárias àquelas de países onde prevalece o Estado Democrático de Direito. Outrossim, foi possível notar 
que, dentre os países da OCDE, aqueles governados por mulheres obtiveram melhores resultados em comparação com aqueles governados por homens. Um exemplo gritante disso pode ser obtido quando comparamos a taxa de mortalidade dos Estados Unidos com a da Alemanha, por exemplo.

Não é coincidência que os governos femininos tenham obtido melhores resultados no combate à pandemia. Os dados apontam para uma capacidade exemplar das mulheres em lidarem com situações emergenciais e de crise econômica, social e sanitária. Como apontam os estudos analisados no presente artigo, os estereótipos de gênero são falsos, e a discriminação sexual, sem nenhum fundamento além da ignorância. Mulheres são amplamente capazes de liderar países, inclusive, fazendo políticas públicas mais atentas às minorias sociais; manejando situações de risco; tomando decisões racionalmente e obtendo resultados exemplares como chefes de Estado. Está na hora de quebrar o teto de vidro.

\section{A mulher e os espaços de poder}

A relação existente entre o sexo feminino com os espaços de poder foi, durante muito tempo, vista como antagônica. Mas, como veremos a seguir, essa conclusão não resta, verdadeiramente, embasada em dados biológicos ou advindos de um direito natural (apesar de serem esses usados como uma - falsa - justificativa) mas, sim, em decorrência de uma perspectiva social patriarcal, com a imposição de um papel de gênero às mulheres, correspondendo, a este, uma divisão sexual do trabalho.

Apesar da conquista paulatina de diversos direitos por parte das mulheres, através de uma verdadeira luta ao longo dos anos, nota-se sua histórica exclusão das esferas de poder e tomada de decisão. E, não obstante tenham sido firmados diversos tratados e conferências internacionais que tratam desse tema, ${ }^{1}$ a existência formal desses direitos não assegura às mulheres participação equitativa nos espaços de poder, que permanecem com escassa presença feminina na política formal. (REZENDE, 2015, p. 3).

Diante disso, a Declaração da Plataforma de Ação de Beijing, de 1995, ao apresentar a noção de transversalidade, demanda que a perspectiva de gênero seja aplicada a todas as áreas das políticas públicas para que se caminhe no sentido da superação das desigualdades entre homens e mulheres. ${ }^{2}$

\footnotetext{
1 Sobre a ausência de correlação imediata entre a existência de normas que garantam direitos e sua garantia efetiva àqueles apresentados como seus destinatários, confira: Herrera Flores (2009, p. 23-28).

2 Rezende (2015, p. 3-4). A Plataforma de Ação da IV Conferência Mundial sobre a Mulher, realizada em 1995, em Beijing, tem como objetivo o empoderamento de todas as mulheres.
} 
Isso porque a perspectiva de gênero é fundamental para a constituição das relações sociais de sexo, pautadas por construções sociais - e não por um destino biológico (KERGOAT, 2009, p. 67) -, as quais têm por consequência a exclusão das mulheres da cidadania e sua caracterização como minoria social, ${ }^{3}$ e formam a base ontológica para a diferenciação política e social. (SCOTT, 2005, p. 20-21). As relações sociais de sexo ademais estão indissocialmente relacionadas à divisão sexual do trabalho, a qual adquire formas próprias em cada sociedade mas, em geral, tem por característica principal a destinação prioritária da esfera produtiva aos homens e da esfera reprodutiva às mulheres e, consequentemente, com a ocupação, pelos homens, das funções de forte valor social agregado (políticas, religiosas, militares, etc.). (KERGOAT, 2009, p. 67).

A divisão sexual do trabalho ${ }^{4}$ é pautada por dois princípios, legitimados pela lógica naturalista: (KERGOAT, 2009, p. 68) i) o da separação, segundo o qual existem trabalhos de homens e outros de mulheres; e ii) o da hierarquização, segundo o qual um trabalho feito por um homem vale mais do que outro feito por uma mulher. (KERGOAT, 2009, p. 67). Ademais, cabe pontuar que a divisão sexual do trabalho tem uma base material, e não puramente ideológica, e que se baseia em uma relação de poder e de dominação. (KERGOAT, 2009, p. 71).

Acerca dos efeitos negativos gerados pela divisão sexual do trabalho, Firmino, Silva e Viana afirmam:

Os efeitos perversos de tal processo de diferenciação, desde o nascimento, podem ser percebidos na concentração de mulheres nas profissões relacionadas ao cuidado, na baixa presença de mulheres nos espaços de poder de decisão, nas já observadas diferenças salariais entre homens e mulheres, na maior taxa de desocupação das mulheres, na invisibilidade da violência doméstica, na jornada dupla de trabalho feminino, entre outros. (FIRMINO; SILVA; VIANA, 2015, p. 5-6).

A literatura acerca do tema, representada por autores como Abreu de Meirelles; Firmino, Silva e Viana; e Melo, Nastari e Massula, indica a divisão sexual do trabalho e o teto de vidro como os fatores causais da ausência de mulheres nos espaços

\footnotetext{
3 Scott (2005, p. 18-19). Os eventos que determinam que minorias são minorias o fazem através da atribuição do status de minoria a algumas qualidades inerentes ao grupo minoritário, como se essas qualidades fossem a razão e também a racionalização de um tratamento desigual. (...) Processos de diferenciação social produzem exclusões e escravizações que são então justificadas em termos de biologia ou de raça.

4 Essa noção foi primeiramente utilizada pelos etnólogos para designar uma repartição "complementar" das tarefas entre homens e mulheres nas sociedades que estudavam. Lévi-Strauss fez dela o mecanismo explicativo da estruturação da sociedade em família. Mas as antropólogas feministas foram as primeiras que lhe deram um conteúdo novo, demonstrando que traduzia não uma complementaridade de tarefas, mas uma relação de poder dos homens sobre as mulheres (KERGOAT, 2009, p. 67).
} 
de poder e de tomada de decisão. O segundo desses conceitos será analisado no próximo tópico. Quanto à divisão sexual do trabalho, a Plataforma de Ação da IV Conferência Mundial sobre a Mulher, reforçando o que aqui fora dito anteriormente, indicou-a como uma das causas da sub-representação das mulheres nos espaços de poder, uma vez que aumenta os custos de participação feminina na arena política, reforçando a existência de estereótipos, atitudes e práticas discriminatórias, os quais atuam como barreiras à presença de mulheres em partidos políticos, órgãos governamentais, sindicatos e em cargos de direção no setor privado. (REZENDE, 2015, p. 3-4).

Mostra-se relevante, neste ponto, empreender uma breve análise da progressão histórica dos direitos das mulheres frente à divisão sexual do trabalho. Começamos tal análise pela época da Revolução Francesa, quando a igualdade foi anunciada como um princípio geral, uma proposta de que todos os indivíduos seriam considerados iguais para os propósitos de participação política e representação legal. Todavia, a cidadania fora negada para algumas categorias de pessoas, sendo que, dentre elas, constavam as mulheres que tinham como justificativa ao impedimento à participação política seus cuidados com as crianças e seus deveres domésticos. (SCOTT, 2005, p. 15). Neste sentido, a fala do jabonino Pierre-Gaspard Chaumette, citado por Scott:

Desde quando é decente ver as mulheres abandonarem os pios cuidados de suas casas, os berços de seus filhos, para virem a espaços públicos, discursarem nas galerias, nos foros do Senado? Foi aos homens que a natureza confiou os cuidados domésticos? Foi a nós que ela deu seios para amamentar nossas crianças? (LEVY; APPLEWHITE; JOHNSON, 1979, p. 219 apud SCOTT, 2005, p. 15).

Como demonstra Scott, no período das revoluções democráticas, as mulheres foram consideradas como outsiders políticas devido à diferença sexual, e foi então que, na França, o feminismo surgiu como um protesto contra a exclusão das mulheres da política (SCOTT, 2005, p. 20-21), devido à tomada de consciência acerca de uma opressão específica: havia se tornado coletivamente "evidente" que uma enorme massa de trabalho era realizada gratuitamente pelas mulheres; um trabalho invisível e não feito não para si, mas para os outros. Tratava-se dos trabalhos domésticos. (KERGOAT, 2009, p. 67). Apesar da igualdade formal e universal de direitos, prevista na Declaração do Homem e do Cidadão, as mulheres estavam excluídas da possibilidade de serem detentoras de uma gama de direitos. ${ }^{5}$ No que diz respeito, especificamente, à ocupação dos espaços de poder,

5 Scott $(2005$, p. 11). Olympe de Gouges, uma das primeiras feministas que viveu durante a Revolução Francesa, escreveu, como forma de protesto, a Declaração dos Direitos da Mulher e da Cidadã, em 1791, na qual argumentava que todos os direitos dos homens, enumerados pelos revolucionários em 1789, também pertenciam às mulheres. 
tem-se que as mulheres foram socializadas para ver a política como um jogo masculino, um jogo inconsistente com os papéis de gênero atribuídos a elas. (PALMER, 2010. p. 3).

Nesse sentido, qualquer ocupação, por mulheres, de papéis sociais que não condissessem com o papel de gênero atribuído a elas dentro de uma perspectiva machista e paternalista, geraria represálias sociais. ${ }^{6}$ Um exemplo disso é trazido por Palmer e Simon, quando retratam que mulheres que frequentavam a faculdade, nos Estados Unidos, na década de 1940, relataram ter sido advertidas sobre parecer muito espertas e tirar notas altas, uma vez que a exibição de inteligência colocava em risco seu status social no campus. Ademais, constataram as autoras que as mulheres também eram lembradas, geralmente por seus pais e irmãos, de que seguir uma carreira reduziria suas perspectivas de casamento e maternidade. (PALMER, 2010, p. 3).

Foi após a Segunda Guerra Mundial que as mulheres passaram a compor maior índice da força de trabalho no mercado, em decorrência da escassez de mão de obra. Por exemplo, nos EUA, o número de mulheres casadas que trabalhavam saltou de 14,7\% para 21,7\%. (PALMER, 2010, p. 4). Todavia, o quadro de inserção das mulheres na política demorou muitos anos mais para começar a progredir. Até 1975, 48\% dos entrevistados em uma pesquisa conduzida pelo National Opinion Research Center concordaram que "a maioria dos homens se adapta melhor emocionalmente à política do que a maioria das mulheres". (PALMER, 2010, p. 4). O ato de uma mulher entrar na arena eleitoral requeria não apenas coragem política, mas também social, dado que seria certo que sofreria represálias sociais.

Ainda hoje, a presença de mulheres na política é uma exceção, sendo a liderança feminina em cargos públicos mais propensa a ser moderada pelo contexto sociocultural. Em tópico posterior, observaremos como está, hoje, em nível mundial, a ocupação desses cargos por mulheres. Todavia, sabendo-se já do prospecto negativo dessa constatação, cabe indicar que a implementação de programas que visem à mudança dos estereótipos de gênero a nível social é uma das formas para combater essa desigualdade da representação política. (STAJKOVIC; SERGENT, 2020, p. 780).

São vários os fatores internos, externos, interpessoais e organizacionais que impedem o avanço das mulheres na liderança. Destacam-se as barreiras comportamentais

\footnotetext{
6 Cf. Kirkpatrick (1974, p. 15). Ainda segundo a citada autora, como os homens, as mulheres ganham status pelo desempenho eficaz e responsável de papéis culturalmente sancionados. Qualquer esforço para desempenhar papéis atribuídos pela cultura ao sexo oposto provavelmente resultará em uma perda de status na escala de status específico do sexo. Os valores nos quais se espera que as mulheres se concentrem são: afeto, retidão, bem-estar; as habilidades relevantes para a busca desses valores são aquelas associadas a nutrir, servir e agradar uma família e comunidade: cuidar da casa, adornos pessoais, preparar e servir comida, cuidar dos doentes, confortar os abatidos, ajudar e agradar um marido, cuidando e educando os jovens. Além disso, presume-se que essas atividades consumirão todo o tempo das mulheres, que executálas bem é um trabalho tanto de tempo integral quanto para a vida toda.
} 
e atitudinais encontradas nos ambientes organizacionais. Ademais, em nível mundial, tem-se que as mulheres fazem, em média, três vezes mais trabalho não remunerado do que os homens. Às mulheres é atribuído um papel de cuidadoras, seja da casa, dos filhos, dos idosos ou dos enfermos. (JAIN, 2020, p. 30). E, durante a pandemia de COVID-19, esse papel foi ainda mais reforçado (JAIN, 2020, p. 31), como será abordado adiante, no tópico III.

2. A naturalização de um padrão baseado em uma estrutura patriarcal e machista e como o teto de vidro tem influência na manutenção desse padrão

Os baixos percentuais de mulheres que ocupam estruturas de decisão e poder não são o resultado de suas escolhas individuais, mas, sim, as consequências de arranjos estruturais sociais que reforçam a desigualdade existente entre homens e mulheres e, dentro do grupo mulheres, entre aquelas que apresentam uma interseccionalidade de fatores que aumentam sua condição de minoria social, como a raça e a classe social. (FIRMINO; SILVA; VIANA, 2015, p. 6).

Para ilustrar e melhor explicar a limitação existente, que impede as mulheres e outras minorias de alcançarem posições proeminentes de liderança, foi criado, na década de 1970, o conceito de teto de vidro. De acordo com essa teoria, há uma segregação vertical ou, em outras palavras, um afunilamento hierárquico (FIRMINO; SILVA; VIANA, 2015, p. 6), relacionado à ascensão das mulheres em suas carreiras, em decorrência de barreiras invisíveis que podem ser simplificadas, no caso das mulheres, por uma única característica: sua condição de mulher. (JAIN, 2020, p. 30).

Apesar da existência de uma aparente igualdade de oportunidades na progressão de carreira, proporcionada por uma série de proteções legais formais, segundo as quais qualquer pessoa qualificada poderia alcançar os altos cargos, dentro de uma perspectiva meritocrática que sugeriria a obsolescência da teoria do teto de vidro, não apenas as barreiras individuais ainda persistem (PIETRANGELO, 2020), como se fazem bastante presentes no cotidiano corporativo e político, com a inibição sistemática e estrutural do acesso das mulheres aos cargos mais altos da hierarquia profissional e política. (CAMPOS, 2019, p. 41-70).

Dentro desse contexto, as mulheres não são consideradas como iguais concorrentes para um trabalho, já que são aconselhadas e direcionadas a assumirem cargos de tarefas secundárias, ligados aos cuidados de outras pessoas, muitas vezes, e nos quais possam "acomodar" suas atividades profissionais com suas obrigações perante a família. Ibarra, Ely e Kolb citam, em seu artigo, um estudo da psicologia, segundo o qual a maioria das mulheres não conhece a influência da discriminação de gênero em suas vidas, tomando seus efeitos como uma experiência geral (vivenciada por homens e 
mulheres). (IBARRA, 2013). A falta de consciência sobre o fenômeno do teto de vidro é um dos fatores, portanto, que impedem as mulheres de conseguirem lutar contra ele para atingir seu pleno potencial em suas carreiras. (JAIN, 2020, p. 30). É importante destacar que o fenômeno do teto de vidro afeta não apenas a realidade de trabalho das mulheres, mas também sua saúde e bem-estar. Como demonstra Ann Pietrangelo, sintomas como sensação de isolamento, dúvidas e insegurança quanto à própria capacidade, ressentimento e raiva foram observados em mulheres que foram submetidas a um tratamento condizente com o previsto pela teoria do teto de vidro. (PIETRANGELO, 2020).

Mesmo quando as mulheres conseguem, finalmente, chegar ao topo da carreira (o que é uma exceção no quadro geral), suas trajetórias mostram-se muito mais turbulentas do que as dos homens, tendo em vista que sofrem impactos negativos por serem mulheres em seus cotidianos, enquanto os impactos de "ser homem" são, majoritariamente, positivos no âmbito profissional. (CAMPOS, 2019, p. 41-70). Dentre as barreiras decorrentes da desigualdade de gênero impostas às mulheres, notam-se o preconceito e a discriminação, a existência de uma cultura organizacional sexista, a prevalência de modelos de liderança masculinos, a dupla jornada de trabalho e a infraestrutura inadequada. (FIRMINO; SILVA; VIANA, 2015, p. 61-62). As mulheres, ainda, sofrem provações mais intensas e frequentes que os homens, tendo que fazer mais do que eles para "provarem seu valor", ${ }^{7}$ sendo que são julgadas mais duramente do que eventuais candidatos homens seriam. (MOSCHKOVICH, 2013). Mesmo quando passam por esses testes, ainda hoje, as mulheres, no geral, não são tão bem remuneradas quanto os homens, existindo uma grande diferença salarial, pautada pelo sexo, nos mais diversos setores do mercado. (PEW RESEARCH CENTER, 2015).

Muitas vezes, em decorrência da falta de mulheres que ocupem posições elevadas de modo a configurar uma representatividade para as outras mulheres, estas dependem do apoio de uma rede masculina de contatos para ganhar aceitação em seu ambiente de trabalho. (JAIN, 2020, p. 30). No que diz respeito ao âmbito político, tem-se comprovado que a presença de candidatas do sexo feminino encoraja mais mulheres a, em eleições póstumas, também concorrerem.

Todavia, infelizmente, o processo de escalada, pelas mulheres, às posições mais elevadas de destaque político e corporativo ainda precisa, em muito, desenvolverse. Em 2015 foi constatado que $85 \%$ dos executivos e membros de conselho dos Estados

Pew Research Center (2015). Disponível em: https://www.pewsocialtrends.org/2015/01/14/women-andleadership/. Acesso em: 25 set. 2020. "[A]bout four-in-ten Americans point to a double standard for women seeking to climb to the highest levels of either politics or business, where they have to do more than their male counterparts to prove themselves (...) According to a Pew Research Center 2014 survey on women and leadership, 4 in 10 Americans said there's a double standard for women who want to rise to the highest levels of business or politics". 
Unidos eram homens brancos. (EGAN, 2015). Dados mais antigos, mas que, ainda, estão bastante próximos da realidade atual, apontam que, em 2003, o número de mulheres na alta administração das empresas do ranking Fortune 1000 estava entre 7 e 9\%, de acordo com um relatório da Federal Glass Ceiling Commission dos EUA. (FRITSCHER, 2017).

Mesmo quando as mulheres chegam às posições de poder, raramente conseguem liderar por muito tempo. É o que se extrai dos relatórios realizados pelo Fórum econômico Mundial em 2014 (WORLD ECONOMIC FORUM, 2014) e 2016 (WORLD ECONOMIC FORUM, 2016), segundo os quais 38\% das nações estudadas tiveram uma mulher chefe de governo ou de estado por pelo menos um ano nos últimos cinquenta anos (o que corresponde a 56 nações, de um total de 146), mas que, em 31 desses países, as mulheres governaram por menos de cinco anos e, em 10 deles, apenas por um ano. Tem-se, ainda, que, incluindo a Suíça, 60\% dos países atualmente sob liderança feminina estão na Europa. Cabe notar, também, que os EUA e seus vizinhos tiveram pouco ou nenhum tempo sob a liderança feminina. Enquanto os EUA e o México nunca tiveram uma mulher como chefe do executivo, a primeira e única primeira-ministra do Canadá serviu por apenas quatro meses. (GEIGER; KENT, 2017).

3. Uma análise dos papéis de gênero e da divisão sexual do trabalho dentro do contexto da pandemia da COVID-19

Nos tópicos anteriores, pudemos observar, em um quadro geral, quais são os fatores que se apresentam como barreiras internas, externas, interpessoais e organizacionais que impedem o avanço das mulheres para atingir posições de liderança. Com o surgimento da pandemia da COVID-19, não obstante, houve um agravamento dessa situação, antes já severa. Desta feita, entre as consequências da pandemia, destacase o aumento significativo entre as disparidades de gênero, de forma que problemáticas enfrentadas pelas mulheres no contexto pré-pandêmico foram acentuadas, como a violência doméstica, o abuso sexual, a desigualdade salarial, a dupla jornada de trabalho, a insegurança econômica e a segregação nos espaços produtivos e de tomada de decisão, com um reforço ainda maior à divisão sexual do trabalho.

A partir da constatação de que o novo coronavírus poderia ser facilmente transmitido por meio do contato humano, políticas de isolamento social, como a quarentena, passaram a ser incentivadas e adotadas ao redor do mundo, com o intuito de retardar a disseminação do vírus e, consequentemente, achatar a curva de contaminados. Nesse 
contexto, surgiram diversos movimentos ${ }^{8}$ marcados pelo bordão "Stay Home", 9 além de eventos globais como o "One World: Together at home" (ONE, 2020), promovido pela Organização Mundial da Saúde (OMS) e pela Global Citizen em apoio aos profissionais da saúde que atuavam na área de frente do combate à pandemia.

Entretanto, para muitas mulheres, a obrigação de ficar em casa, estipulada pela maioria dos governos locais e nacionais, significou verdadeira exposição a uma situação de insegurança e, muitas vezes, risco de vida, dado que o ambiente doméstico, muitas vezes, representa local de violências, abusos e assédios. (BRADBURY-JONES; ISHAM, 2020). Nesse sentido, diversos relatórios apontaram para um aumento dos casos de violência doméstica durante a pandemia ao redor do globo, uma vez que, em muitos casos, as mulheres se viram confinadas, dentro de casa, com seus agressores ou violadores, sem a possibilidade de pedir socorro. ${ }^{10}$

Estima-se que, em certos países membros da OECD, houve um aumento alarmante no número desses casos. Nos Estados Unidos, por exemplo, houve um aumento de 20 a 30\% em determinadas regiões. (KOFMAN; GARFIN, 2020). Igualmente, na Espanha, constatou-se um aumento de $20 \%$ nos casos de violência doméstica no início da pandemia. (BRADBURY-JONES; ISHAM, 2020, p. 2.047). No mesmo patamar chegaram os números do Reino Unido, onde houve um aumento de $25 \%$ das ligações para a $U K$ Domestic Violence Helpline. (BRADBURY-JONES; ISHAM, 2020, p. 2.047). Outros países, como o Brasil, atingiram números alarmantes, com um aumento de $40 \%$ a $50 \%$ dos casos de violência doméstica. (BRADBURY-JONES; ISHAM, 2020, p. 2.047). Em um quadro geral, dados fornecidos pelo Fundo de População das Nações Unidas (UNFPA), junto de seus parceiros Avenir Health e Johns Hopkins University, nos EUA, e Victoria University, na Austrália, apontaram um aumento de $20 \%$ na violência doméstica sofrida durante os três primeiros meses de quarentena em todos os 193 estados membros da ONU

8 O cerne da política que fundou tais movimentos, encontra-se na ideia de restrição da locomoção por parte da população, que deveria restar na situação de confinamento, saindo de casa apenas para atividades consideradas essenciais como compras para abastecimento em supermercados, compra de medicamentos ou para trabalhar caso fosse impossível a execução da atividade laboral no modelo home office.

9 Pode-se citar, aqui, o movimento "Stay home today, \#traveltomorrow", promovido pela Organização Mundial do Turismo com intuito de conter o turismo temporariamente até o retorno do setor de maneira sustentável (UNITED NATIONS. World Tourism Organization. Stay home today, \#traveltomorrow. Madrid, 6 April 2020. Disponível em: https://www.unwto.org/news/stay-home-today-traveltomorrow. Acesso em 29 de setembro de 2020); ainda, pudemos também acompanhar o movimento "Stay home, save lives", propagado na Europa e traduzido para as 25 línguas dos países membros da União Europeia. Disponível em: https://cdt.europa.eu/en/news/stay-home-save-lives-25-languages. Acesso em: 29 de setembro de 2020.

10 Nesse cenário, as mulheres são submetidas, de forma forçosa e, durante tempo integral, a relações abusivas, sem que possam abandonar seus lares ou recorrer a interações sociais e a redes de apoio, o que possibilita aos homens perpetradores de violência uma ampla liberdade para cometerem abusos, sem que exista qualquer tipo de repressão social ou estatal. 
(os números levam em consideração os altos níveis previstos de casos subnotificados). (FORD, 2020).

Conforme apontado pelo Índice de Instituições Sociais e Gênero do Centro de Desenvolvimento, de 2019, da OCDE, a violência doméstica está diretamente relacionada a sua aceitação social. (RAMOS, 2020, p. 13). Assim, ocorre, normalmente, em um cenário em que a figura masculina repressora se encontra livre de qualquer possibilidade de repressão, podendo agir dentro da normatividade da masculinidade patriarcal, socialmente aceita, para exercer poder e controle sobre as mulheres, inclusive por meio da violência física ou sexual. Ademais, nos níveis individual e familiar, os perpetradores de violência podem querer reafirmar seu controle e expressar suas frustrações causadas pela situação pandêmica por meio de episódios crescentes de violência. (RAMOS, 2020, p.12-13).

Também no contexto da COVID-19 observou-se, em diversos países, a sobrecarga de mulheres que passaram a despender mais tempo em tarefas domésticas (RAMOS, 2020, p. 12-13), e em cuidados com os filhos, havendo um aumento significativo da dupla jornada de trabalho como resultado das políticas de quarentena e isolamento social. Afinal, tais políticas levaram ao fechamento de escolas, creches, serviços públicos de educação e cuidado infantil, gerando um trabalho extra no âmbito familiar que, em sua grande maioria, teve de ser assumido pelas mulheres (DEL BOCA; OGGERO; PROFETA; ROSSI, 2020. p. 2), em vez de ocasionar uma maior exposição de homens a tarefas historicamente atribuídas à figura feminina. (DEL BOCA; OGGERO; PROFETA; ROSSI, 2020. p. 5). Além de cuidados com as crianças, foi observado que recaem sobre mulheres as obrigações de cuidado com familiares doentes, deficientes e idosos, mesmo quando empregados. ${ }^{11}$ Esses fatos evidenciaram como crises sanitárias e sociais podem afetar de maneira distinta homens e mulheres em razão dos papéis de gênero, reforçando as desigualdades de gênero em muitas dimensões. ${ }^{12}$

Esse resultado mostra-se preocupante, pois revela a manutenção das disparidades de gênero na sociedade atual, mesmo após anos de luta dos movimentos feministas. Demonstram, ainda, como barreiras histórica, culturais e sociais são fundamentais para a manutenção da estrutura patriarcal e o reforço aos papéis de gênero. Dessa forma, o cenário articulado pela pandemia da COVID-19 colabora para manutenção

\footnotetext{
11 Del Boca, Oggero, Profeta e Rossi (2020. p. 4-5). "Available data for European OECD countries show that employed women are $50 \%$ more likely than employed men to report that they regularly take care of ill, disabled or elderly adult relatives".

12 Inclusive, nesse sentido, alguns estudos sobre o contexto italiano confirmaram a situação de sobrecarga de mulheres que tiveram que lidar com suas atividades remuneradas regulares, equilibrando-as com tarefas domésticas e familiares adicionais introduzidos pela pandemia da COVID-19. Em termos estatísticos, pesquisas indicam que $68 \%$ das mulheres italianas reportaram aumento do trabalho doméstico como consequência do lockdown, enquanto apenas $40 \%$ dos homens italianos reportaram o mesmo (PRIOLA, 2020, p. 2).
} 
e reforço das barreiras tratadas pela teoria do teto de vidro, dado que potencializa a exclusão das mulheres dos espaços produtivos e decisionistas.

As barreiras representadas pelo trabalho doméstico e de cuidado familiar, aumentadas no período da pandemia, ademais, estão afetando desproporcionalmente mulheres e as impedindo de desenvolver suas habilidades de trabalho e estudo. Assim, enquanto as submissões de periódicos, em geral, aumentaram durante a pandemia, a proporção dessas submissões de autoria de mulheres, na maioria dos casos, diminuiu. (OLESCHUK, 2020, p. 3). Desta feita, conclui-se que a pandemia está prejudicando desproporcionalmente a produtividade de mulheres acadêmicas em comparação com homens desse meio. (OLESCHUK, 2020, p. 4).

Ainda, cabe analisar de que forma a pandemia afetou as mulheres em termos de subsistência e rendimentos. Para isso, é importante ter em mente que uma grande parte das mulheres sobrevivem da economia informal, sobretudo em países subdesenvolvidos, tornando-as mais suscetíveis ao desemprego no contexto pandêmico. Um outro fator que colabora para o atual aumento da taxa de desemprego entre as mulheres é o fato de que elas se concentram em determinados setores da economia que têm potencial de serem mais afetado pela pandemia da COVID-19. (RAMOS, 2020, p. 6).

De acordo com relatório da OECD, as mulheres dos países membros encontram-se, normalmente, concentradas no setor público e nos setores de saúde e educação e, além disso, representam a maioria dos empregados nos setores como o de serviços de alimentação e bebidas, (em que representam 53\% dos empregados); serviços de alojamento (onde constituem 60\% da força de trabalho); e varejo (que conta, em média, com $62 \%$ de mulheres entre seus colaboradores), os quais foram especialmente afetados pela pandemia. (RAMOS, 2020, p. 7).

Ademais, as disparidades econômicas associadas às diferenças de gênero tendem a ser agravadas, no contexto da pandemia, considerando que entre os membros da OECD, o nível de pobreza entre as mulheres é maior do que entre os homens, o que pode ter diversas explicações, entre as quais as diferenças salariais e a dificuldade de inserção no mercado de trabalho em razão das responsabilidades atribuídas às mulheres dentro da divisão sexual do trabalho. (RAMOS, 2020, p. 7-8).

Conforme apontado anteriormente, mulheres encontram-se concentradas no setor da saúde e de cuidados, chegando a representar $70 \%$ da força de trabalho na área da saúde. (AGHAJANIAN, 2020, p. 4). As mulheres estão na vanguarda da luta contra o coronavírus, representando dois terços da força de trabalho da saúde em todo o mundo. (AGHAJANIAN, 2020, p. 1-3). Contudo, apesar de representar a linha de frente do combate à COVID-19, as mulheres encontram-se sub-representadas em cargos de 
tomada de decisão na política e na economia, ${ }^{13}$ bem como em cargos relacionados à gestão da COVID-19 e seus efeitos. ${ }^{14}$ A mesma sub-representação ocorre, entre os membros da OCDE, dentro da área da saúde, na qual as mulheres ocupam apenas uma minoria dos cargos de liderança. (RAMOS, 2020, p. 3).

Tal fato influencia diretamente a tomada de decisões de políticas públicas, que, assim, se tornam completamente negligentes quanto às implicações diversas do vírus, considerando o marcador de gênero na população. Assim, a condição de sub-representação feminina em espaços de planejamento e tomada de decisão, não só reforça o papel da mulher como cuidadora, fortalecendo as barreiras sociais e culturais que sustentam, historicamente, a desigualdade de gênero, como, também, impede o desenvolvimento de políticas públicas que poderiam reduzir os impactos da pandemia sobre as mulheres.

Na estrutura social vigente, em decorrência do fenômeno do teto de vidro, as mulheres são bloqueadas no acesso a cargos de tomada de decisão. Dessa forma, políticas são necessárias para lidar com a falta de igualdade de acesso e de oportunidades, o que passa pela reforma dos processos de contratação e promoção a cargos superiores, que se baseiam, principalmente, em uma meritocracia patriarcal. Além de políticas positivas de inserção de mulheres em posições de poder, são necessárias políticas públicas de suporte e oferecimento de serviços públicos acessíveis, que poderiam ajudar as mulheres a gerenciar o trabalho doméstico e se concentrar no desenvolvimento de suas carreiras e vidas produtivas, laborais e acadêmicas. (PRIOLA, 2020, p. 7).

Nesse sentido, algumas medidas são elencadas pela OECD, a fim de reduzir as disparidades de gênero que foram destacadas no contexto da COVID-19. Entre essas medidas é possível elencar o oferecimento de creches públicas para pais que trabalham em serviços essenciais; apoio financeiro para trabalhadores que precisam se afastar; políticas de acesso a benefícios dirigidos a famílias de baixa renda, em particular pais solteiros; desenvolvimento de políticas para apoiar vítimas de violência de gênero, como oferecer serviços de auxílio eletrônicos suficientemente seguros que facilitem as denúncias na época de lockdown e adotar medidas contra a aceitação social da violência doméstica, como campanhas de conscientização sobre como esse problema afeta diversas mulheres em época de confinamento. (RAMOS, 2020, p. 14-24).

13 O Canadá teve o maior percentual de mulheres em comitês de nível nacional com 52\%, e o Brasil teve o menor, com 3,7\%. 74\% dos países tinham menos de um terço do sexo feminino em cargos governamentais nacionais. (FUHRMAN; RHODES, 2020, p. 3 e 32).

14 Ramos (2020, p. 3). Ainda sobre isso, confira Aghajanian e Page (2020, p. 2). "Há lacunas notáveis no número de mulheres em órgãos de tomada de decisão do COVID-19 em nível nacional ou internacional (...). Isso se estende aos países do Norte Global, bem como aos países do Sul Global. (...) Uma análise acerca da força de trabalho em saúde global, publicada pela OMS, revelou que as mulheres representavam $70 \%$ da força de trabalho de saúde da linha de frente, mas apenas $25 \%$ dos líderes no setor de saúde". 
Ademais, a adoção de medidas que garantam o acesso de mulheres a espaços decisionais nesse período são essenciais, tendo em vista que as mulheres compõem a linha de frente de combate à COVID-19, enquanto representantes da maioria no setor da saúde. Tal acesso pode ser garantido por meio de políticas públicas que aumentem o papel e número de mulheres atuantes no setor. Além disso, seria favorável, para tais objetivos, a alteração da estrutura de organismos nacionais e internacionais, a exemplo da Organização Mundial da Saúde (OMS), referência na condução de políticas de saúde a nível mundial, que revela uma lacuna crítica de especialistas em gênero, o que influenciam diretamente na tomada de decisões e na governança de surtos causados por doenças infecciosas. (AGHAJANIAN; PAGE, 2020, p. 2).

Contudo, a execução de políticas públicas não se mostra suficiente para alterar as desigualdades de gênero existentes, em uma análise a longo prazo, sendo necessário, sobretudo, a adoção de medidas destinadas a alterar as barreiras históricas, sociais e culturais solidificadas ao longo do tempo, de modo a mudar substancialmente as expectativas sociais em relação à figura masculina e ao papel paterno, reconhecendoos como iguais responsáveis pelo cuidado infantil e trabalho doméstico. Afinal políticas podem minimizar as desigualdades existentes, mas não eliminam a fonte dessas desigualdades. (PRIOLA, 2020, p. 7-8).

4. Governanças femininas e masculinas, diferenças na abordagem de políticas públicas e seus resultados.

Como estudado anteriormente, de acordo com a teoria do teto de vidro, as mulheres encontram mais dificuldades em alcançar posições de liderança e de tomada de decisão do que os homens, em decorrência de barreiras invisíveis que as impedem de subir em suas carreiras após um certo patamar. Essas barreiras são impostas socialmente como resultado de uma divisão sexual do trabalho, das relações sociais de sexo e das desigualdades de gênero que, ainda hoje, persistem em nossa sociedade.

As dificuldades encontradas pelas mulheres em alcançar cargos de liderança, muitas vezes, são justificadas por falácias discriminatórias, com a disseminação de estereótipos que não correspondem à realidade.

A pandemia COVID-19, contudo, além da trágica calamidade que causou à saúde pública global e à economia de muitos países, engendrou uma situação política na qual se tornou evidente a incompatibilidade dos estereótipos de gênero com a real capacidade de governança e de tomada de decisões das mulheres. Isso porque, dentre os dez países que obtiveram o menor número de mortes em decorrência da pandemia, oito são governados por mulheres. (ALMEIDA FILHO, 2020). 
Esse dado pode ser explicado pelo fato de que quando as mulheres alcançam cargos de poder e gestão, tendem a tomar decisões assertivas, demonstrando ampla competência para administrar, até mesmo, situações adversas. Essa premissa ficou evidente em diversos estudos que analisaram os resultados obtidos por países governados por mulheres no cenário da pandemia.

Esses países têm conseguido responder de forma rápida e eficaz à disseminação do vírus, adotando e coordenando políticas para controlar e reduzir seus impactos na população. A esse respeito, Henley e Roy dizem: "Da Dinamarca, Finlândia, Alemanha, Islândia e Noruega na Europa, até Taiwan e Nova Zelândia na Ásia-Pacífico, as mulheres líderes superaram seus colegas homens ao tomarem ações decisivas informadas por organizações humanitárias e princípios cooperativos" (HENLEY, 2020) e, também, de acordo com Garikipati e Kambhampati: “O desempenho das mulheres líderes na pandemia do COVID-19 oferece uma experiência global única na gestão de crises nacionais, onde várias questões, incluindo a eficácia da liderança, podem ser examinadas entre os países". (GARIKIPATI; KAMBHAMPATI, 2020, p. 3).

Os países com governança feminina não apenas representam a maioria dos países com os menores coeficientes de mortalidade, mas, também, assumem as primeiras posições de países com os menores coeficientes de contaminação, com base em parâmetros coletados no primeiro semestre de 2020 , como pode ser visto na tabela abaixo:

Tabela 1 - Indicadores epidemiológicos da Pandemia COVID-19 por gênero do Chefe de Governo em países selecionados.

\begin{tabular}{|c|c|c|c|c|}
\hline País & Chefe de Governo & $\begin{array}{l}\text { Incidência p/ } \\
10 \text { mil hab }\end{array}$ & $\begin{array}{r}\text { Letalidade } \\
\%\end{array}$ & $\begin{array}{r}\text { Mortalidade } \mathrm{p} / \\
\text { mi hab }\end{array}$ \\
\hline \multicolumn{5}{|c|}{ Países com os 10 maiores coeficientes de mortalidade } \\
\hline Bélgica & Sophie Wilmés & 45,38 & 16,32 & 740,40 \\
\hline Espanha & Pedro Sánchez & 47,81 & 11,84 & 566,32 \\
\hline Itália & Sergio Mattarella & 36,10 & 13,93 & 502,71 \\
\hline Reino Unido & Boris Johnson & 31,70 & 14,67 & 465,30 \\
\hline França & Emmanuel Macron & 21,27 & 18,95 & 403,07 \\
\hline Suécia & Stefan Löfven & 25,66 & 12,42 & 318,83 \\
\hline Holanda & Mark Rutte & 24,73 & 12,79 & 316,43 \\
\hline Irlanda & Michael Higgins & 46,09 & 6,35 & 292,17 \\
\hline EUA & Donald Trump & 39,56 & 6,02 & 233,17 \\
\hline Suíça & Simonetta Sommaruga & 34,85 & 5,08 & 176,90 \\
\hline \multicolumn{5}{|c|}{ Países com os 10 menores coeficientes de mortalidade } \\
\hline Dinamarca & Mette Frederiksen & 17,81 & 5,10 & 90,12 \\
\hline Alemanha & Angela Merkel & 20,19 & 4,37 & 87,95 \\
\hline
\end{tabular}




\begin{tabular}{|l|r|r|r|r|}
\hline Finlândia & Sanna Marin & 10,60 & 4,51 & 46,92 \\
\hline Noruega & Erna Solberg & 14,88 & 2,64 & 39,29 \\
\hline Islândia & Katrín Jakobsdóttir & 52,78 & 0,56 & 29,30 \\
\hline Grécia & Katerina Sakellaropoulou & 2,60 & 5,57 & 14,39 \\
\hline Nova Zelândia & Jacinda Ardern & 2,37 & 1,84 & 4,36 \\
\hline Coréia do Sul & Chung Sye-kyun & 2,12 & 2,35 & 4,99 \\
\hline China & Xi Jinping & 0,58 & 5,52 & 3,22 \\
\hline Taiwan & Tsai Ing-wen & 0,18 & 1,36 & 0,25 \\
\hline
\end{tabular}

Legenda: Governante mulher: fundo azul; Governante homem: fundo rosa

Referência: Almeida Filho (2020).

Na mesma linha, estudos mencionados por Aghajanian (FIORAMONTI, 2020 apud AGHAJANIAN; PAGE, 2020, p. 2) revelaram a eficácia dos governos femininos no combate à COVID-19. Nesse sentido, a análise de trinta e cinco países concluiu que (i) os países liderados por mulheres registraram seis vezes menos mortes por COVID-19 do que os países liderados por homens; (ii) foram mais eficientes e ágeis no achatamento da curva da doença, com ápice seis vezes menor do que o observado em países liderados por homens. (AGHAJANIAN; PAGE, 2020, p. 2).

É importante destacar que nos Estados governados por mulheres, o número de óbitos pela COVID-19 foi menor tanto em termos numéricos quanto percentuais, ${ }^{15}$ além de apresentarem, em média, menores taxas de contágio registradas. (GARIKIPATI; KAMBHAMPATI, 2020, p. 4). Diante dessa realidade, é fundamental buscar os motivos para que esses países tenham apresentado melhores resultados em termos de contaminação e morte.

Dentre as respostas encontradas estão os fatores associados às boas políticas adotadas pelos governos das mulheres, como as medidas rápidas e eficientes para conter o novo vírus, bem como os discursos proferidos por representantes de governo femininas em face da pandemia, os quais foram realizados na forma de comunicação direta com a população, de forma clara e transmitindo confiança.

Um dos primeiros fatores de interesse revela-se na posição feminina tendente a não assumir riscos em assuntos relacionados a perdas humanas - como sugeria o contexto da pandemia -, assim, mulheres, enquanto governantes, tendem a não assumir riscos que coloquem em xeque vidas e o bem-estar humano, diferentemente de homens

15 Stajkovice e Sergent (2020, p. 776). Ainda, nesse sentido: "It is clear that female-led countries have fared better in terms of absolute number of COVID-cases and deaths, with male-led countries having nearly double the number of deaths as female-led ones. (...) Our results so far confirm that women-led countries performed better in terms of the number of COVID-deaths experienced and also (though less significantly) in the number of cases". (GARIKIPATI; KAMBHAMPATI, 2020, p. 7-10). 
na mesma posição. (GARIKIPATI; KAMBHAMPATI, 2020, p. 2). Também cabe destacar que, quando se trata de questões econômicas, mulheres tendem a assumir mais riscos se comparadas aos homens, pois não seria essa sua prioridade, frente a uma situação de caos sanitário. Mostram-se, portanto, mais humanitárias em sua tomada de decisão, revelando que existem diferenças de gênero na tomada de decisões em cenários de risco e incerteza. (GARIKIPATI; KAMBHAMPATI, 2020, p. 11-12).

Assim, em países sob liderança feminina, medidas de quarentena e isolamento social foram adotadas mais rapidamente, potencializando seus efeitos na contenção da disseminação do novo vírus, o que, mais tarde, refletiria menores taxas de letalidade. Nesse sentido, vale destacar o exemplo do governo alemão, o qual, sob decisão de Angela Merkel, adotou rapidamente o lockdown no início da pandemia de maneira preventiva, apesar de possuir um eficiente sistema de saúde, assumindo eventuais efeitos negativos sobre a economia do país. (PIAZZA; DIAZ, 2020, p. 7-8).

Outras pesquisas comparativas apontam para a adoção de uma postura orientada para a resolução de problemas ou tarefas, por parte de governos masculinos, e para a adoção de uma postura interpessoal em governos femininos. (GARIKIPATI; KAMBHAMPATI, 2020,p. 14). Com isso, tem-se também que homens têm maior propensão ao exercício de governos autoritários, enquanto mulheres tendem a exercer governos mais democráticos, participativos e cooperativos. (GARIKIPATI; KAMBHAMPATI, 2020, p. 14). Nesse sentido, torna-se bastante ilustrativo o governo da Noruega, no qual a primeira ministra, Erna Solberg, organizou campanhas de esclarecimento da população a respeito da COVID-19 e acerca das medidas de quarentena e distanciamento social. (ALMEIDA FILHO, 2020).

Outro padrão de comportamento identificado em mulheres que ocupam posições de liderança se refere a atitudes empáticas e cooperativas. Como já dito, lideranças femininas tendem a seguir uma linha democrática de governo, privilegiando a troca de informação, ideias e experiências (STAJKOVIC; SERGENT, 2020, p. 773), o que colabora para identificação e instituição de políticas mais efetivas na resolução de problemas sociais, sanitários e econômicos, por exemplo. Tal comportamento cooperativo foi observado no contexto dos governos locais liderados por mulheres nos Estados Unidos, os quais passaram a compartilhar entre si medidas e políticas bem-sucedidas de combate à COVID-19, as quais, inclusive, divergiam do discurso negligente adotado pelo governo federal. Nesses governos locais, medidas de isolamento e confinamento foram adotadas de maneira rápida e preventiva, apesar de eventuais efeitos colaterais na economia, o que 
resultou em melhores resultados no índice de contágio a nível local, em contraposição aos catastróficos resultados atingidos a nível nacional pelos EUA. ${ }^{16}$

Ademais, grande parte dos governos nacionais femininos mostram-se socialmente engajados em mitigar o agravamento de disparidades sociais no contexto de COVID-19, o que está relacionado com as próprias histórias de dificuldades enfrentadas pelas mulheres que alcançam posições de poder, dado que apresentam uma maior compreensão das dificuldades que prejudicam minorias. (FUNK, 2020, p. 4).

A postura cooperativa assumida por governos femininos tende a ser especialmente importante no cenário de pandemia, pois leva à incorporação de medidas de cooperação no plano internacional, apoiando o multilateralismo. Esse movimento é essencial para a resolução de problemas transfronteiriços, assim como para a coordenação de ações e políticas orientadas a resolver problemáticas comuns aos planos nacionais. No contexto da pandemia tem-se que muitas dessas problemáticas locais que atingem as mulheres foram agravadas, tais como abuso, exploração, violência doméstica, desigualdades sociais e econômicas causadas pela diferença de gênero. (TAKSA, [2020]. p. 3).

Além de padrões comportamentais baseados no gênero influenciarem significativamente na condução de lideranças e governos, tem-se que esses padrões se refletem, também, no discurso dos líderes políticos, o que interfere diretamente na aderência populacional às políticas e medidas propostas e adotadas.

Diante de situações negativas, há reações psicológicas distintas com base no gênero. Assim, enquanto homens tendem a reagir com raiva e imprudência diante de cenários e resultados futuros incertos, mulheres, por sua vez, tendem a reagir com cautela. (STAJKOVIC; SERGENT, 2020, p. 776). Esse padrão comportamental feminino associado a outros já explicados, como aqueles fundados na empatia e cooperação, refletem-se diretamente no discurso de líderes mulheres, que acabam por passar maior confiança aos governados.

Não por outra razão, estudos demonstram que mulheres tendem a ser preferidas como líderes em momentos de crise, tendo em vista sua capacidade de entender as necessidades de todos. Assim, uma possível razão para que as medidas de isolamento social e de confinamento tenham sido pronta e voluntariamente atendidas por residentes de países liderados por mulheres reside na confiança e empatia transmitidas em seus discursos. Ademais, essas líderes se mostram engajadas com a saúde, bem-estar e

16 Funk (2020, p. 3). "In this sense, it is appropriate to highlight the speech of Lightfoot, mayor of Chicago, criticizing the federal approach to the pandemic: 'I'm a Black woman in America. Nobody's coming to rescue me. I've got to get it for myself, and I feel the same way with what we need to do here in our city' In the same sense follows the speech of Bottoms, mayor of Atlanta: 'We've got to be prepared for this regardless of what the federal preparations and the state preparations will be"'. 
segurança dos cidadãos. Isso justifica o maior sucesso das políticas de isolamento social e confinamento nesses Estados quando comparados a outros países liderados por homens que adotaram as mesmas medidas e obtiveram piores resultados. ${ }^{17}$

Um bom exemplo de sucesso no enfrentamento à COVID-19 é a Dinamarca, que, apesar do fato de ser um dos países com maior qualidade de vida, apresentou, também, outros fatores que explicam o sucesso das políticas de contenção da COVID-19. Entre esses fatores, podem-se citar: (i) seu posicionamento geográfico isolado; (ii) baixa desigualdade social, o que possibilita igual adoção de medidas de prevenção por parte de grande parcela da população; (iii) distribuição demográfica de 5,8 milhões de pessoas espalhadas por $42,9 \mathrm{~km}^{2}$, com densidade demográfica de 137 pessoas por $\mathrm{km}^{2}$, o que favoreceu o isolamento geográfico; (iv) disposição de um sistema de saúde gratuito e acessível a toda a população; (v) cultura que tem baixa prática de contato físico, como abraços, aperto de mãos ou beijos somente manifestados em relação a parentes próximos. (OLAGNIER; MOGENSEN, 2020, p. 10-11).

Todavia, esses fatores culturais, sociais, demográficos e geográficos não são suficientes para explicar, isoladamente, o sucesso das medidas de isolamento social e confinamento para contenção da COVID-19 nesse país, tendo em vista que na Suíça, país que possui elementos similares, os resultados de contenção dos casos de COVID-19 não foram tão bem sucedidos. Assim, a adoção das medidas de confinamento e isolamento social logo no início da expansão da COVID-19, de maneira preventiva, associadas ao discurso da líder desse país, o qual estimulou a adoção dessas medidas (que tiveram grande aderência populacional) pode explicar a diferença entre os resultados obtidos por esses dois países. (OLAGNIER; MOGENSEN, 2020, p. 10-11).

Para além do exemplo dinamarquês, a Alemanha também aparece como destaque no combate ao coronavírus. Desde o começo da pandemia, a Alemanha procurou adotar medidas ativas de contenção do vírus, assumindo uma postura preventiva de modo a evitar o risco de perdas humanas, confirmando o traço contrário a riscos, característico de lideranças femininas. Nesse sentido, Angela Merkel desenvolveu uma resposta embasada cientificamente, promovendo testes generalizados, transparência na divulgação de dados, políticas rigorosas de distanciamento social, restrição de viagens, além de apelos ao público para o efetivo cumprimento dessas medidas. (PIAZZA; DIAZ, 2020, p. 7-8).

Além disso, a resposta fundamentada de Merkel confirma outro traço comum aos governos femininos quando comparados aos masculinos, que diz respeito

17 Stajkovic e Sergent (2020, p. 776). "Women governors who issued early stay-at-home orders had fewer COVID-19 deaths in their states than men governors who issued the same orders. This suggests that state residents perhaps responded differently depending on whether a man or woman governor issued this order, as manifested by lives saved. (...) stay-at-home orders issued by women governors were potentially delivered with greater empathy and confidence compared to men governors". 
à tendência das mulheres a admitirem suas limitações, consultando especialistas sempre que necessário, a fim de adotar políticas públicas mais assertivas e efetivas.

Não obstante, a implementação de tais políticas não foi pacífica, tampouco imune a críticas. Enquanto alguns afirmavam que dentro de um cenário extremo o confinamento poderia durar cerca de dois anos, o que seria completamente insustentável para a economia alemã, outros, como Hans-Juergen, presidente da Corte Constitucional Alemã, afirmavam que era impossível e, diametralmente contrário às liberdades civis, a adoção de medidas de isolamento social e confinamento, que criavam, em última instância, um governo autoritário, antidemocrático e de vigilância. (JAIN, 2020, p. 52).

Apesar das reações críticas e negativas, Merkel não deixou de sustentar as medidas cientificamente embasadas, preferindo colocar em risco a economia às vidas humanas. O equilíbrio entre medidas de confinamento e proteção econômica teve de ser realizado por todos os países de alguma forma. Na Alemanha, a escolha pelas medidas rigorosas de isolamento social, logo no começo da pandemia, ajudou na contenção da COVID-19, resultando em baixos índices de infectados e mortos, quando comparados a outros países da Europa Central. Entretanto, cabe salientar que as medidas de isolamento e confinamento, apesar de rigorosas e cientificamente embasadas, não foram adotadas de forma isolada, pois Merkel garantiu que uma série de medidas para mitigar os efeitos sobre a economia, entre as quais o implemento de auxílios emergenciais para microempresas e trabalhadores autônomos, e auxílio a grandes empresas por meio de um fundo de estabilização econômica. (JAIN, 2020, p. 53).

Diante do exposto, conclui-se que as governanças femininas se mostraram, dentro do recorte temporal da presente pesquisa, bem-sucedidas no combate à COVID-19 por uma associação de fatores psicológicos e estratégicos que resultaram no controle do vírus com um saldo de menos mortos e infectados, quando comparados a governos cujo dirigente é do sexo masculino. Entre os padrões identificados em governos liderados por mulheres, é possível ressaltar a tendência a evitar riscos relativos à perda humana, preferindo arriscar a economia, sem, contudo, desconsiderar os impactos sobre tal setor. Essa característica influenciou na adoção, rápida e preventiva, de medidas de confinamento e isolamento social, em atendimento ao determinado por especialistas da área da saúde, evitando que o vírus se espalhasse exponencialmente, de modo a permitir sua contenção nesses países.

Além disso, o discurso empático, resiliente e realista de líderes mulheres passou confiança ao público que, voluntariamente, aderiu às políticas de isolamento, garantindo, assim, o sucesso de tais medidas. $\mathrm{O}$ sucesso em termos de vidas humanas associado a perdas contornáveis no campo econômico, pelo menos no primeiro momento da pandemia, demonstra como mulheres têm condições de ocupar espaços de poder, desenvolvendo lideranças racionais e estratégicas, sobretudo, em momentos de crise. 
Conclusão

Através da pesquisa que gerou o presente artigo, foi possível constatar que, em decorrência das circunstâncias únicas geradas pela pandemia da COVID-19, os modelos de governança feminina e masculina foram colocados à prova, possibilitando um quadro comparativo de sua eficiência frente a situações emergenciais complexas, que envolvem questões ligadas à saúde pública, à educação, e à economia. Por meio dos dados analisados, foi possível concluir que, não apenas mulheres são tão capazes quanto homens em exercerem, de forma eficaz, prudente e bem-sucedida, cargos de governança e tomada de decisão como, também, mostraram-se ainda mais competentes que seus parceiros diplomáticos masculinos.

Isso se dá, não como poderiam supor alguns teóricos, por tratar-se de uma situação de calamidade sanitária, enquanto seriam as mulheres cuidadoras naturais dentro de uma perspectiva naturalista, mas, sim, pelo fato das mulheres se mostrarem líderes mais empáticas, abertas à cooperação com outros governantes, responsáveis, cautelosas, que requerem opiniões de experts para tomar decisões fundamentadas e que priorizam a vida do que a economia.

Os melhores resultados obtidos por países governados por mulheres não são mera coincidência, são o resultado de políticas mais responsáveis, adotadas por governos mais comunicativos e democráticos, onde medidas preventivas foram implementadas logo no início da pandemia, em detrimento de possíveis consequências negativas econômicas, com o objetivo de poupar o maior número de vidas possível.

A pandemia da COVID-19, portanto, apesar de ter ocasionado efeitos devastadores por todo o mundo, permitiu o esclarecimento de uma questão que, há muito, restava ainda obscura pela falta de oportunidades: mulheres são tão hábeis quanto os homens para ocupar cargos de liderança e de tomada de decisões e, não é possível mais admitir que as barreiras invisíveis, impostas pelo teto de vidro, continuem impedindo que as mulheres cresçam em suas carreiras, atinjam cargos elevados, posições de destaque, poder e comando. Os estereótipos de gênero, mais uma vez e, de forma bastante explícita, se mostraram falsos.

Para que o teto de vidro seja quebrado, é preciso não apenas o estabelecimento de políticas públicas a nível nacional, respeitando-se os padrões culturais de cada país, de forma a evitar uma imposição hegemônica de padrões culturais, de valores ou de governo, mas, também, a mudança estrutural de órgãos nacionais e internacionais, para que mais mulheres sejam incorporadas aos espaços de poder e possam, assim, formar uma rede de representatividade para que outras mulheres também possam lá chegar. Ademais, estereótipos de gênero precisam ser extirpados da sociedade, de forma a não dar mais 
espaço para a perpetuação de um padrão machista e patriarcal organizacional. Para isso, é importante o amplo estabelecimento de políticas de gênero nos mais diversos âmbitos.

São Paulo, outubro de 2020.

\section{Referências}

AGHAJANIAN, Lina; PAGE, Ella. COVID-19 and the participation of women and women's rights organizations in decision-making. K4D: Knowledge, evidence and learning for development, Brighton, UK, 10 July 2020. Disponível em: https://opendocs.ids.ac.uk/opendocs/bitstream/ handle/20.500.12413/15532/850_COVID-19_and_the_participation_of_women_in_decision_ making.pdf?sequence $=1 \&$ isAllowed $=\mathrm{y}$.

ALMEIDA FILHO, Naomar de. Mulheres no comando e no poder: efetividade, afetividade e credibilidade política contra a pandemia. ABRASCO: Associação Brasileira de Saúde Coletiva, Rio de Janeiro, 12 maio 2020. Disponível em: https://www.abrasco.org.br/site/noticias/especialcoronavirus/mulheres-no-comando-e-no-poder-efetividade-afetividade-e-credibilidade-politicacontra-a-pandemia-artigo-de-naomar-de-almeida-filho/48127/. Acesso em: 28 set. 2020.

BRADBURY-JONES, Caroline; ISHAM, Louise. The pandemic paradox: the consequences of COVID-19 on domestic violence. Journal of Clinical Nursing, Hoboken, v. 29, n. 13-14, p. $2.047-$ 2.049, July 2020. Disponível em: https://onlinelibrary.wiley.com/doi/epdf/10.1111/jocn.15296. Acesso em: 29 set. 2020.

CAMPOS, Isabelle Oglouyan de. Mulheres na Academia: desigualdades de gênero no corpo docente da Faculdade de Direito da Universidade de São Paulo. 2019. Trabalho de Conclusão de Curso (Bacharelado em Direito) - Faculdade de Direito, Universidade de São Paulo, São Paulo, 2019.

DEL BOCA, Daniela; OGGERO, Noemi; PROFETA, Paola; ROSSI, Maria Cristina. Women's work, housework and childcare, before and during the COVID-19. Bonn: IZA Institute of Labor Economics. June 2020. (Discussion Paper Series). Disponível em: http://ftp.iza.org/dp13409.pdf.

EGAN, Matt. Still missing: female business leaders. CNN Business, Atlanta, 24 March 2015. Disponível em: https://money.cnn.com/2015/03/24/investing/female-ceo-pipeline-leadership/. Acesso em: 29 set. 2020.

FIRMINO, Camila Rocha; SILVA, Filipe Hagen Evangelista da; VIANA, Pedro Henrique Pina Cabral. Desigualdades de gênero no serviço público federal. In: CONGRESSO CONSAD DE GESTÃo PÚBLICA, 8., 2015, Brasília. Anais [...]. Brasília, Conselho Nacional de Secretários de Estado da Administração, 2015.

FORD, Liz. 'Calamitous': domestic violence set to soar by $20 \%$ during global lockdown. The Guardian, London, 28 Apr. 2020. Disponível em: https://www.theguardian.com/globaldevelopment/2020/apr/28/calamitous-domestic-violence-set-to-soar-by-20-during-globallockdown-coronavirus. Acesso em: 30 set. 2020. 
FRITSCHER, Lisa. The glass ceiling effect and its impact on women. everyday health. Everyday Health, New York, 15 Nov. 2017. Disponível em: https:/www.everydayhealth.com/womens-health/ glass-ceiling-effect-its-impact-on-women/._Acesso em: 30 set. 2020.

FUHRMAN, Sarah; RHODES, Francesca. Where are the women? The conspicuous absence of women in COVID-19 response teams and plans, and why we need them. Geneva: Care International, June 2020. Disponível em: https://insights.careinternational.org.uk/media/k2/attachments/CARE_ COVID-19-womens-leadership-report_June-2020.pdf. Acesso em: 30 set. 2020.

FUNK, Kendall D. Local responses to a global pandemic: women mayors lead the way. Politics \& Gender, Cambridge, July 2020. DOI: https://doi.org/10.1017/S1743923X20000410. Disponível em: https://www.cambridge.org/core/journals/politics-and-gender/article/local-responses-to-aglobal-pandemic-women-mayors-lead-the-way/28311D9DC2651D9B843A1318DFD2009B.

GARIKIPATI, Supriya; KAMBHAMPATI, Uma. Leading the fight against the pandemic: does gender 'really' matter? Social Science Research Network, Rochester, 3 June 2020. Disponível em: https://ssrn.com/abstract=3617953 ou em: http://dx.doi.org/10.2139/ssrn.3617953. Acesso em: 28 de set. 2020 .

GEIGER, Abigail W.; KENT, Lauren. Number of women leaders around the world has grown, but they're still a small group. Pew Research Center, Washington, DC, 8 Mar. 2017. Disponível em: https:/www.pewresearch.org/fact-tank/2017/03/08/women-leaders-around-the-world/. Acesso em: 25 set. 2020 .

HENLEY, Jon; ROY, Eleanor Ainge. Are female leaders more successful at managing the coronavirus crisis? The Guardian, London, 25 Apr. 2020. Disponível em: https://www.theguardian. com/world/2020/apr/25/why-do-female-leaders-seem-to-be-more-successful-at-managing-thecoronavirus-crisis. Acesso em: 30 set. 2020.

HERRERA FLORES, Joaquín. A (re) invenção dos direitos humanos. Tradução de: Carlos Roberto Diogo Garcia, Antônio Henrique Graciano Suxberger e Jefferson Aparecido Dias. Florianópolis: Fundação Boiteux, 2009.

IBARRA, Herminia; ELY, Robin J.; KOLB, Deborah M. Women rising: the unseen barriers. Harvard Business Review, Brighton, Sept. 2013. Disponível em: https://hbr.org/2013/09/womenrising-the-unseen-barriers. Acesso em: 27 set. 2020.

JAIN, Priyadarshini. Women leadership in Indian higher education sector: the realities, challenges and future directions post COVID 19 pandemic. MAT Journals, Ghaziabad, v. 1, n. 1, Jan.-June 2020 .

KERGOAT, Danièle. Divisão sexual do trabalho e relações sociais de sexo. In: HIRATA, Helena; LABORIE, Françoise; LE DOARÉ, Hélène; SENATIER, Danièle (org.). Dicionário crítico do feminismo. São Paulo: Editora Unesp, 2009.

KIRKPATRICK, Jeane J. Political woman. New York: Basic Books, 1974. 
KOFMAN, Yasmin B.; GARFIN, Dana Rose. Home is not always a haven: the domestic violence crisis amid the COVID-19 Pandemic. Psychological Trauma: Theory, Research, Practice, and Policy, Washington, DC, v. 12, n. S1, p. 199-201, Aug. 2020.

MOSCHKOVICH, Marília Bárbara Fernandes Garcia. Teto de vidro ou paredes de fogo? Um estudo sobre gênero na carreira acadêmica e o caso da UNICAMP. 2013. 159 p. Dissertação (Mestrado) Faculdade de Educação, Universidade Estadual de Campinas, Campinas, SP, 2013.

OLAGNIER, David; MOGENSEN, Trine H. The Covid-19 pandemic in Denmark: big lessons from a small country. Cytokine \& Growth Factor Reviews, London, v. 53, p. 10-12, June 2020.

OLESCHUK, Merin. Gender equity considerations for tenure and promotion during COVID-19. Canadian Review of Sociology, Mississauga, v. 57, n. 3, p. 502-515, Aug. 2020.

ONE world: together at home. Portal Global Citizen, [s. l.], 18 Apr. 2020. Disponível em: https:// www.globalcitizen.org/en/connect/togetherathome/. Acesso em: 30 set. 2020.

PALMER, Barbara; SIMON, Dennis. Breaking the political glass ceiling: women and congressional elections. 2. ed. Abingdon: Routledge, 2010.

PEW RESEARCH CENTER. Women and leadership: public says women are equally qualified, but barriers persist, Washington, DC, Jan. 2015. Disponível emt: https://www.pewsocialtrends. org/2015/01/14/women-and-leadership/. Acesso em: 25 set. 2020.

PIAZZA, Kelly Senters; DIAZ, Gustavo. Light in the midst of chaos: COVID-19 and female political representation. World Development, Amsterdam, v. 136, Dec. 2020.

PIETRANGELO, Ann. The impacts of the glass ceiling effect on people. Healthline, San Francisco, CA, 16 June 2020. Disponível em: https://www.healthline.com/health/mental-health/glass-ceilingeffect. Acesso em: 29 set. 2020.

PRIOLA, Vincenza; Pecis, Lara. Missing voices: the absence of women from Italy's Covid-19 pandemic response. Gender in Management, Bingley, Special Issue, Sept. 2020. Disponível em: https://www.emerald.com/insight/content/doi/10.1108/GM-07-2020-0218/full/pdf?title=missingvoices-the-absence-of-women-from-italys-covid-19-pandemic-response.

RAMOS, Gabriela. Women at the core of the fight against COVID-19 crisis. Organisation for Economic Co-operation and Development-OECD, Paris, 1 Apr. 2020. Disponível em: http://www. oecd.org/coronavirus/policy-responses/women-at-the-core-of-the-fight-against-covid-19-crisis553a8269/\#section-d1e1834. Acesso em: 29 set. 2020.

REZENDE, Daniela Leandro. Mulher no poder e na tomada de decisões. Brasília: IPEA Instituto de Pesquisa Econômica Aplicada, 2015. Disponível em: https:/www.ipea.gov.br/retrato/ pdf/190215_tema_g_mulher_no_poder_e_na_tomada_de_decisoes.pdf. Acesso em: 28 ago. 2020.

SCOTT, Joan W. O enigma da igualdade. Revista Estudos Feministas, Florianópolis, v. 13, n. 1, p. 11-30, jan./abr. 2005. 
STAJKOVIC, Alexander D.; SERGENT, Kayla. Women's leadership is associated with fewer deaths during the COVID-19 Crisis: quantitative and qualitative analyses of United States governors. Journal of Applied Psychology, Washington, DC, v. 105, n. 8, p. 771-783, 2020.

TAKSA, Lucy. What the world needs now...! Women leaders, multilateralism and internacional co-operation, [2020]. Disponível em: https://www.mq.edu.au/_data/assets/pdf_file/0004/1007095/ cwf-cidp-taksa-women-leaders-multilateralism-and-cooperation.pdf.

WORLD ECONOMIC FORUM. The global gender gap report 2014. Cologny: [s. n.], 2014. Disponível em: http://www3.weforum.org/docs/GGGR14/GGGR_CompleteReport_2014.pdf. Acesso em: 29 set. 2020.

WORLD ECONOMIC FORUM. The global gender gap report 2016. Cologny: [s. n.], 2016. Disponível em: http://www3.weforum.org/docs/GGGR16/WEF_Global_Gender_Gap_ Report_2016.pdf. Acesso em: 28 set. 2020. 